\title{
Tecnologías del Aprendizaje y el Conocimiento como estrategias en la formación de los docentes de la Escuela Normal Superior de Cúcuta, Colombia*
}

\author{
[Versión en Español]
}

Learning and Knowledge Technologies as Strategies in Training

Teachers of La Normal Superior School, Cúcuta, Colombia

As tecnologias da aprendizagem e do conhecimento como estratégias na formação de professores da Escola Superior Normal de Cúcuta, Colômbia

Recibido septiembre 27 de 2018. Aceptado julio 24 de 2019.

Para citar este artículo:

Díaz-Guecha, Leonardo-Yotuhel; Márquez-Delgado, Rodolfo-Alfonso (2020). Tecnologías del Aprendizaje y el Conocimiento como estrategias en la formación de los docentes de la Escuela Normal Superior de Cúcuta, Colombia. Ánfora, 27(48), 19-42. DOI:

https://doi.org/10.30854/anfv27.n48.2020.667 Universidad Autónoma de Manizales. ISSN 0121-6538/ e-ISSN 2248-6941
Leonardo-Yotuhel Díaz-Guecha** https://orcid.org/0000-0003-2619-5742

Colombia

Rodolfo-Alfonso Márquez-Delgado***

https://orcid.org/0000-0003-4501-074X

Venezuela

\section{Resumen}

Objetivo: generar elementos teóricos que fundamenten las estrategias de las Tecnología del Aprendizaje y Conocimiento TAC en el proceso de formación de los docentes de la Escuela Normal Superior

\footnotetext{
* Este artículo es un avance de la Tesis Doctoral en Ciencias de la Educación que realiza el autor principal de este artículo en la Universidad Pedagógica Experimental Libertador de Venezuela -Instituto de Mejoramiento Profesional del Magisterio, Extensión Académica San Cristóbal, Táchira.

** Magíster en Educación. Especialista en Gerencia Informática. Abogado. Profesor del Programa de Derecho Universidad Simón Bolívar-Cúcuta. Estudiante de Doctorado en Ciencias de la Educación. Correo:I.diaz01@unisimonbolivar.edu.co

*** Postdoctorado en Gerencia de la Educación Superior. Doctor en Ciencias Administrativas. Magíster en Gerencia de Empresas. Especialista en Evaluación Educativa. Licenciado en Educación mención Matemática de la Universidad Pedagógica Experimental Libertador. Profesor de la Universidad del Táchira, Venezuela. Correo: upeltachira@yahoo.es
} 
María Auxiliadora de Cúcuta Colombia. Metodología: se desarrolló bajo el enfoque cualitativo, sustentado en el paradigma interpretativo introspectivo vivencial, con el fin de develar las estrategias pedagógicas (TAC) donde se realiza la investigación, es decir, a partir de la realidad de los docentes, sus experiencias, percepciones, vivencias. En este estudio participaron 10 docentes formadores de formadores de la Escuela Normal Superior de Cúcuta, Colombia, seleccionados del Programas de Formación Complementaria de la Institución. Para la recolección de los datos se utilizó la entrevista focalizada con preguntas abiertas. Resultados: se generaron tres elementos teóricos que fundamentan las estrategias TAC: Formación por competencias para la consolidación de las TAC; Pensamiento crítico y aprendizaje basado en problemas mediante el uso de la estrategia TAC y La construcción del conocimiento en el proceso de enseñanza-aprendizaje. Conclusiones: para consolidarse en el aula, las estrategias TAC deben construirse sobre elementos teóricos que permitan a los docentes redimensionar su actividad para ser gestores y mediadores en el uso y aplicación de estas tecnologías emergentes.

Palabras-clave: Estrategias TAC; Método de enseñanza; Formadores; Aprendizaje y Conocimiento; Competencias.

\section{Abstract}

Objective: to provide theoretical elements to lay the foundations of the strategies for Learning and Knowledge Technology (LKT) in the process of teacher training of La Normal Superior School María Auxiliadora, Cúcuta, Colombia. Methodology: this research was conducted under the qualitative approach, based on the experiential introspective interpretative paradigm, in order to research pedagogical strategies $(L K T)$. This means it is based on the teachers' reality, their experiences, perceptions, and experiences. This study comprised of ten teacher trainers from La Normal Superior School, Cúcuta, Colombia, who were selected from the Institutional Complementary Training Programs. Data was collected through focused interviews with open questions. Results: three theoretical issues arose from the LKTs: Training through competences for the consolidation of LKT strategies; Critical thinking and problem-based learning using LKTs, and the construction of knowledge in the teaching-learning process. Conclusions: in order to be strengthened within the classroom, LKTs must be built up on theoretical issues, to allow teachers to resize their activity to become managers and mediators in the use and application of these emerging technologies.

Keywords: LKT strategies; Teaching Method; Trainers; Learning and Knowledge; Competences. 


\section{Resumo}

Objetivo: gerar elementos teóricos que apóiem as estratégias da Tecnologia da Aprendizagem e do Conhecimento TAC no processo de formação de professores da Escola Superior Normal Maria Auxiliadora de Cúcuta Colômbia. Metodologia: foi desenvolvida sob a abordagem qualitativa, com base no paradigma interpretativo introspectivo experiencial, a fim de revelar as estratégias pedagógicas (TAC) onde a pesquisa é realizada, ou seja, com base na realidade dos professores, suas percepç̃̃es e experiências. Neste estudo, participaram 10 instrutores da Escola Superior Normal de Cúcuta, Colômbia, selecionados entre os Programas Institucionais de Treinamento Complementar da instituição. A entrevista focada com perguntas abertas foi usada para coletar os dados. Resultados: foram gerados três elementos teóricos que sustentam as estratégias das TAC: Treinamento por competências para a consolidação das TAC; Pensamento crítico e aprendizagem baseada em problemas através do uso da estratégia TAC e da construção de conhecimento no processo de ensinoaprendizagem. Conclusões: para ser consolidadas na sala de aula, as estratégias TAC devem ser construídas sobre elementos teóricos que permitam aos professores redimensionar suas atividades para serem gerentes e mediadores no uso e aplicação dessas tecnologias emergentes.

Palavras-chave: Estratégias TAC; Método de ensino; Formadores; Aprendizagem e conhecimento; Competências. 


\section{Introducción}

En este mundo globalizado, los adelantos tecnológicos y científicos ocurren con vertiginosa velocidad y, por ende, la necesidad de adquirir los conocimientos para una mejor convivencia y calidad de vida. La humanidad, entre el tránsito del siglo pasado y el actual, ha desarrollado múltiples tecnologías informáticas que innovan velozmente los procesos educativos, con la oferta de un mayor poder de información notables avances enriquecedores en los procesos educativos apoyados con las tecnologías.

El presente artículo tiene como objetivo generar elementos teóricos que fundamenten las estrategias TAC en el proceso de formación de los docentes de la Escuela Normal Superior María Auxiliadora de Cúcuta, Colombia. Para ello, se nutre de investigaciones de autores como Simoes (2017), quien considera que "En la actualidad uno de los fenómenos más importantes en la transformación ha sido la introducción de las TIC en todos los ámbitos de la vida” (p. 10) y muy especialmente en el educativo.

Estas investigaciones, como producto de los cambios en la sociedad de la información, han ido más allá con lo que se ha denominado las tecnologías de aprendizaje y el conocimiento (TAC), las cuales están enfocadas en aprender a aprender y al aprendizaje colaborativo, o como lo indican Segura, Candioti y Medina (2007), hacia la actualización permanente del desarrollo de conocimientos, habilidades y destrezas de los estudiantes, mediante un proceso de enseñanza que es continuo, cambiante y se desarrolla mediante la generación de entornos virtuales.

Se trata, entonces, de un cambio de paradigma en los usos formativos de las Tecnologías de la Información y la Comunicación (TIC) de los estudiantes y los docentes, por cuanto ello implica no solo dominar las herramientas sino saber utilizarlas y aplicarlas para la adquisición de conocimientos, que es el objetivo de las TAC. Por ejemplo, Moya-López (2013) plantea que las TAC han abierto un nuevo panorama a los docentes, estudiantes, ambiente educativo e instituciones frente a los cuales se debe estar atento, no solo por su uso formativo, desarrollo de contenidos educativos, sino por las metodologías interactivas y contextualizadas que se apliquen en los procesos de enseñanza-aprendizaje.

La reflexión sobre los planteamientos de Moya-López (2013), suministraron evidencias teóricas a los investigadores para dilucidar acerca de la problemática existente en la Escuela Normal Superior de Cúcuta, Colombia con respecto a la conceptualización que tienen los docentes formadores de formadores sobre el uso de las estrategias TAC en el aula, las cuales son fundamentales para la conducción del proceso de aprendizaje y construcción de conocimientos. 
En otras palabras, en su proceso de formación, el docente debe asumir un cambio del escenario tradicional sobre las TIC hacia otro que requiere transformación de actitudes, motivación, construcción, generación de conocimientos y una adecuada metodología en los procesos de aprendizaje en el aula; sin embargo, se ha detectado, durante conversaciones y observaciones permanentes de los investigadores, que las instituciones educativas, entre ellas, la Escuela Normal Superior de Cúcuta, escenario de la investigación, ha sido lenta para introducir las TAC como estrategias que contribuyan a la formación integral del individuo.

En la institución educativa en la que se hizo este estudio, se encontraron otros nudos problemáticos relacionados con el uso tradicional de estrategias tecnológicas en la formación de los docentes, por cuanto éstas se utilizan para el desarrollo de procesos cognitivos de bajo nivel, como búsqueda de datos, informaciones, eventos y con escasas estrategias pedagógicas que permitan establecer las relaciones con la práctica y la búsqueda de conocimientos.

$\mathrm{Al}$ respecto, Lozano (2011) asevera que "En realidad lo que se plantea es cambiar el aprendizaje de la tecnología por el aprendizaje con la tecnología, enfoque que está orientado totalmente al desarrollo de competencias fundamentales como el aprender a aprender" (p. 1). Esto significa que los docentes, en su proceso formativo no solo deben utilizar las tecnologías por moda sino comprender que éstas les permitirán ayudarlos a pensar, a indagar lo que les interesa aprender; así, puede desechar de manera voluntaria lo que considera de escasa utilidad para que su formación se fundamente en un aprendizaje consciente que emerja de la indagación y el uso de estrategias tecnológicas colaborativas, interactivas y metacognitivas.

Unido a la problemática antes indicada ocurre que, en ocasiones, los docentes en formación, manifiestan conductas tecnofóbicas y sienten rechazo y temor por el uso de las TIC y de los cambios que pueden producirse en el aprendizaje de los estudiantes. Al respecto, Suay (2017) opina que "La nueva tecnología no se ha realizado sin la resistencia por parte de las instituciones y de las personas. Las actitudes tecnofóbicas han estado presentes a lo largo de la historia" (p. 1). De allí, que los docentes se inhiban ante ellas debido, probablemente, a su desconocimiento, lo cual les causa incertidumbre y piensan que no son capaces de utilizarlas; esto ocurre, sobre todo cuando muchos de sus estudiantes son versados en el uso de las TIC como herramientas de obtención de información, aunque no sean usuarios inteligentes y críticos de la información y del proceso de construcción de conocimientos.

La problemática anterior, ha sido ampliamente discutida por investigaciones realizadas por Rosen y Weil (2000), quienes demostraron en un estudio realizado con más de 2000 profesores de 54 centros escolares de 
California que los profesores sentían un alto grado de tecnofobia por el uso de las tecnologías; aun cuando habían sido obligados a realizar cursos de computación, sus actitudes no habían mejorado después de la formación. Esta conclusión rompió con la creencia de que los profesores por aprobar cursos sobre el uso de las herramientas, se interesaban y mejoraban sus actitudes frente a las tecnologías. En esta conclusión, se evidencia, la concepción tradicional de la enseñanza de las TIC la cual se enfocaba al proceso de recibir, recopilar y procesar la información, pero no a transformarla en conocimientos base fundamental para enfrentar los cambios de la sociedad del aprendizaje.

Otro aspecto que es producto de las limitaciones planteadas está en el hecho de que algunos docentes formadores de formadores poseen escasa formación y competencias en el uso de las TIC y más aún de las TAC; así que, en ambos casos, desconocen su significado e importancia para la formación integral del estudiante, lo que trae como consecuencia deserción, desmotivación, poco interés, apatía, lo cual les genera no solo dificultades para asimilar los procesos durante el desarrollo de las actividades en el aula sino hasta rechazo de los estudiantes que serán los futuros maestros para ejercer en el Nivel de Preescolar y Básica Primaria, tanto en Cúcuta como en otros lugares.

En atención a la problemática planteada, en este estudio se genera la siguiente interrogante: ¿Qué elementos teóricos fundamentan las estrategias TAC en el proceso de formación de los estudiantes de la Escuela Normal Superior María Auxiliadora de Cúcuta, Colombia?

\section{Metodología}

La investigación es de enfoque cualitativo sustentada en el paradigma interpretativo introspectivo vivencial, lo cual permitió develar los elementos teóricos que fundamentan las estrategias TAC desde el propio contexto donde se realizó la investigación; es decir, a partir de la realidad de los docentes, sus experiencias, percepciones, vivencias.

Los datos se obtuvieron de las entrevistas realizadas a 10 docentes de la Escuela Normal la Superior María Auxiliadora de Cúcuta (Colombia), quienes fueron seleccionados observando los criterios que indican Rodríguez, Gil y García (1999) en cuanto a la idoneidad, singularidad, disponibilidad y complejidad, características que atribuyen una condición única y reveladora para la comprensión de la realidad estudiada. Se incluyeron además otros aspectos como la antigüedad en la Institución y la comunicación abierta y franca con el investigador. Las entrevistas se realizaron durante los meses de septiembre-diciembre de 2017. Las fases que se desarrollaron en el proceso metodológico fueron: 
a) Revisión de los referentes relacionados con el objeto de estudio.

b) Entrevista a profundidad a los diez (10) docentes de la Escuela Normal Superior de Cúcuta para describir, comprender e interpretar sus vivencias y experiencias en el uso de las estrategias pedagógicas TAC en el proceso de enseñanza-aprendizaje. Las preguntas indagaron principalmente acerca de la formación que poseen los docentes en el uso de las TAC en el aula; el conocimiento que tienen los docentes sobre las estrategias TAC; el uso y aplicación de las estrategias TAC en el aprendizaje.

c) La transcripción de las entrevistas realizadas a los participantes en la investigación permitió tomar lo expuesto en las fuentes originarias y proceder a la segmentación de la información, cuya finalidad fue la búsqueda de emergencias discursivas en los docentes entrevistados, haciendo énfasis en oraciones, frases o expresiones, las cuales facilitaron develar la esencia del mensaje, su sentido y significado.

d) A partir de allí, se derivaron las dimensiones que surgieron de la integración y análisis de los códigos centrales sobre el uso pedagógico de las estrategias TAC; además, tales estrategias se organizaron en componentes: Formativo, Aplicativo y Conceptual los cuales originaron las categorías cuyas nociones permitieron interpretar la realidad y resumir las dimensiones provenientes del proceso de análisis.

e) La triangulación fue la base para el análisis de la información. Para ello, se entrelazaron los datos que se encontraron en la investigación con las bases referenciales conformando el corpus de resultados de la investigación.

f) Posteriormente, se hizo la interpretación, discusión de los resultados para construir los elementos teóricos que fundamentan las estrategias TAC en el proceso de formación de los estudiantes de la Escuela Normal Superior María Auxiliadora de Cúcuta (Colombia).

g) Finalmente, se formularon las conclusiones o ideas reflexivas.

\section{Análisis, organización y sistematización de la información}

La actividad desarrollada en la recolección de la información dio paso a la triangulación como exigencia de los estudios de naturaleza cualitativa. Se trata del proceso que, de acuerdo con Leal (2015), consiste en “...emplear, dentro de un mismo estudio, diversidad de elementos propios de la metodología de la investigación, entre los cuales cabe destacar los métodos, las teorías, las fuentes y los tipos de datos, los informantes y las técnicas de indagación” (p. 20). Por tanto, triangular significa contrastar entre los distintos hallazgos en las actividades 
interpretativas descritas anteriormente. Con la triangulación se pretendió comprobar y confrontar los datos obtenidos en las etapas enunciadas para averiguar la relación o contraste entre las fuentes de datos.

En ese sentido, para Stake (1999), la triangulación tiene como propósito presentar un cuerpo de descripción, conseguir la confirmación, aumentar el crédito de la interpretación; para este autor hay varias estrategias para triangular, una es de la fuente de datos, para interpretar la información suministrada por los participantes, que es lo que se realiza en este estudio; sin embargo, también se recurre a lo que el autor denomina triangulación de la teoría, que no es más que comparar lo obtenido a la luz de los planteamientos teóricos que sustentan el estudio.

Lo realizado, de acuerdo con Martínez (2007), supuso examinar, revisar la información que emana de las variadas fuentes de testimonios. Luego, se procedió a comparar lo recabado; es decir, establecer las semejanzas y diferencias. Después, se efectuó la distinción-sumario, mediante el cual se crea el texto representante de la nueva realidad en investigación, surgida de la asociación y de la divergencia entre las fuentes de datos.

Ahora, según Rodríguez, Gil y Suárez (1996; citados por Martínez, 2007) el procedimiento da lugar al análisis de contenido, con el propósito de avanzar más allá de lo manifestado por los informantes. Por ello, es una actividad para esculcar en lo expresado lo que está más allá de las expresiones pronunciadas y visibilizar desde las palabras, los argumentos teóricos planteados en el estudio. Así, en el proceso investigativo se enfatizó en las actividades realizadas para obtener los datos, en la codificación respectiva, la cual facilita agrupar los datos en categorías, conceptos o constructos, sustentados en las similitudes y diferencias. Todo ello, incidió para definir las categorías emergentes en este estudio, pues siguiendo a Ángel (2011) "El proceso de esta construcción de categorías, pasa por una codificación que el investigador efectúa hasta llegar a la codificación selectiva” (p. 17). A continuación se recoge en la Tabla 1 el proceso aplicado: 
Tabla 1. Análisis, organización, sistematización de la información

\begin{tabular}{|c|c|c|c|c|}
\hline 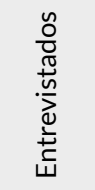 & Códigos centrales & 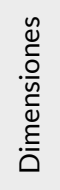 & Categorías & $\begin{array}{c}\text { Elementos } \\
\text { teóricos }\end{array}$ \\
\hline $1,3,4,5$ & $\begin{array}{c}\text { Adquisición de compe- } \\
\text { tencias para conocer los } \\
\text { alcances de las TAC } \\
\text { Desconocimiento de los } \\
\text { fundamentos teóricos de } \\
\text { las TAC como estrategias } \\
\text { Desconozco el significado } \\
\text { de las TAC. Esto es un } \\
\text { modernismo más de las } \\
\text { tecnologías } \\
\text { No me gusta trabajar con } \\
\text { las tecnologías, no tengo } \\
\text { las competencias } \\
\text { No poseo las actitudes } \\
\text { para } \\
\text { utilizar las tecnologías } \\
\text { Siento que son difíciles de } \\
\text { utilizar }\end{array}$ & 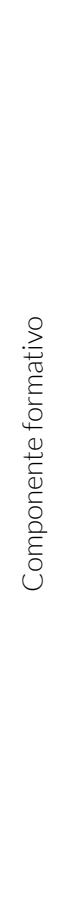 & $\begin{array}{c}\text { Desconocimiento } \\
\text { del término TAC } \\
\text { Conozco algo sobre las } \\
\text { TIC, pero } \\
\text { necesito formación } \\
\text { Creo que las TAC } \\
\text { son estrategias, pero } \\
\text { estoy seguro de que a } \\
\text { veces } \\
\text { pienso que son } \\
\text { técnicas } \\
\text { Son unas herramientas } \\
\text { más de la tecnología } \\
\text { No veo la } \\
\text { diferencia con } \\
\text { respecto a las TIC } \\
\text { Modernismo tecnológico } \\
\text { No las utilizo porque las } \\
\text { desconozco. } \\
\text { Necesito formación }\end{array}$ & $\begin{array}{l}\text { Formación } \\
\text { por competencias } \\
\text { para la consolida- } \\
\text { ción de } \\
\text { las TAC }\end{array}$ \\
\hline
\end{tabular}




\begin{tabular}{|c|c|c|c|c|}
\hline 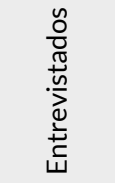 & Códigos centrales & 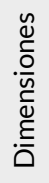 & Categorías & $\begin{array}{c}\text { Elementos } \\
\text { teóricos }\end{array}$ \\
\hline $7,5,6,10$ & $\begin{array}{c}\text { Uso esporádico de las } \\
\text { TIC y menos aún de las } \\
\text { TAC } \\
\text { en el aula } \\
\text { Creo que las TAC son } \\
\text { estrategias que ayudan a } \\
\text { Pensar } \\
\text { Las TIC TAC son conside- } \\
\text { radas herramientas que } \\
\text { facilitan el trabajo en el } \\
\text { aula } \\
\text { Las tecnologías son } \\
\text { útiles en los procesos de } \\
\text { enseñanza como fuente de } \\
\text { información en actividades } \\
\text { del aula } \\
\text { mus } \\
\text { mucha utilidad en el aula } \\
\text { porque garantizan inte- } \\
\text { racciones, colaboración, } \\
\text { intercambio, discusión } \\
\text { pero tengo compañeros } \\
\text { que sí lo han hecho y dicen } \\
\text { que son herramientas } \\
\text { muy interesantes para la } \\
\text { docencia. } \\
\text { Las }\end{array}$ & 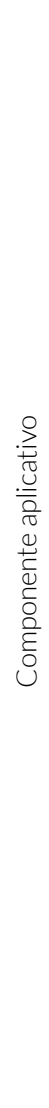 & $\begin{array}{l}\text { Solo utilizo el internet } \\
\text { No se aplicar las TAC en } \\
\text { el quehacer pedagógico } \\
\text { Su uso facilita la actividad } \\
\text { del aula } \\
\text { Son estrategias que se } \\
\text { utilizan para interactuar, } \\
\text { compartir } \\
\text { Son estrategias que } \\
\text { ayudan a la búsqueda de } \\
\text { información } \\
\text { Garantizan interac- } \\
\text { tividad, intercambio, } \\
\text { discusión } \\
\text { Creo pueden utilizar- } \\
\text { se para la solución de } \\
\text { problemas } \\
\text { tegias TAC } \\
\text { Su uso y aplicación } \\
\text { Podría ser útil para el } \\
\text { Desarrollo del pensa- } \\
\text { miento crítico de los } \\
\text { estudiantes } \\
\end{array}$ & $\begin{array}{c}\text { Pensamiento crítico } \\
\text { y aprendizaje ba- } \\
\text { sado en problemas } \\
\text { mediante el uso de } \\
\text { la estrategia TAC }\end{array}$ \\
\hline
\end{tabular}




\begin{tabular}{|c|c|c|c|c|}
\hline 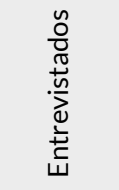 & Códigos centrales & 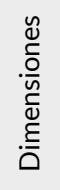 & Categorías & $\begin{array}{c}\text { Elementos } \\
\text { teóricos }\end{array}$ \\
\hline $2,8,9,1,2$ & $\begin{array}{l}\text { Las TAC son herramientas } \\
\text { que permiten aprender } \\
\text { a aprender } \\
\text { Las estrategias TAC y las } \\
\text { TIC, son herramientas } \\
\text { pero las primeras permiten } \\
\text { la construcción del } \\
\text { conocimiento } \\
\text { Las TAC son estrategias } \\
\text { alternativas diferentes } \\
\text { a las TIC que pueden } \\
\text { utilizarse en el aula } \\
\text { Son estrategias emer- } \\
\text { gentes que permiten la } \\
\text { creatividad } \\
\text { Estrategias para la forma- } \\
\text { ción por competencias } \\
\text { Contribuyen a la formación } \\
\text { del individuo } \\
\text { Las TAC son estrategias } \\
\text { para la formación integral }\end{array}$ & 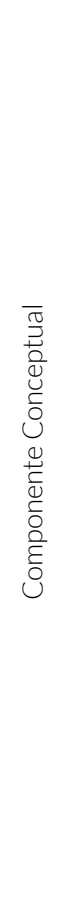 & $\begin{array}{c}\text { Estrategias emergentes } \\
\text { para el quehacer peda- } \\
\text { gógico } \\
\text { Estrategias para apren- } \\
\text { der a aprender } \\
\text { TIC y TAC son herra- } \\
\text { mientas tecnológicas, } \\
\text { pero con objetivos } \\
\text { diferentes } \\
\text { Las TAC favorecen } \\
\text { la creatividady } \\
\text { la formación integral } \\
\\
\text { Las TAC } \\
\text { Son potenciadoras } \\
\text { del proceso } \\
\text { de aprendizaje }\end{array}$ & $\begin{array}{l}\text { La construcción } \\
\text { del conocimiento en } \\
\text { el proceso de ense- } \\
\text { ñanza-aprendizaje } \\
\text { sustentado } \\
\text { en las estrategias } \\
\text { TAC }\end{array}$ \\
\hline
\end{tabular}

Fuente: elaboración propia

\section{Resultados}

Para la compilación e interpretación de los resultados se tomaron en cuenta los objetivos de la investigación, los testimonios de los docentes que participaron las dimensiones y categorías señaladas en la Tabla 1. Las categorías emergentes fueron producto del análisis de las respuestas dadas por los participantes. Éstas se analizan a continuación:

1. El Componente Formativo estuvo orientado hacia las respuestas que, con regularidad, indicaron los entrevistados 1, 3, 4, 5 con respecto al dominio o formación que poseen sobre las TAC. Estos aspectos se rela- 
cionan directamente con los rasgos comunes que identifican a los profesores y se concreta en los modos de actuar frente a las tecnologías y a las estrategias TAC. Un aspecto que estuvo presente en los entrevistados es no poseer las competencias necesarias para analizar, aplicar y argumentar sobre el significado y alcance de las TAC y los basamentos teóricos que las fundamentan. Este componente, una vez reflexionado y analizado, derivó el elemento teórico denominado "Formación por competencias para la consolidación de las TAC".

2. El Componente Aplicativo, se derivó de las respuestas reiteradas de los entrevistados 7, 5, 6, 10. Todos ellos manifestaron que las tecnologías son importantes en el aula porque facilitan el proceso de aprendizaje, la resolución de problemas y el pensamiento crítico-reflexivo. Pese a este planteamiento, indicaban los entrevistados que las utilizan esporádicamente, ya que la institución no les suministra los recursos para desarrollarlas ni cuentan con el ambiente propicio en el aula para implementarlas. Todos coincidieron, sin embargo, en la importancia y alcance de las TAC como estrategias para lograr posibles interacciones, colaboración, intercambio y discusión; sin embargo, en repetidas ocasiones indicaban los docentes que no las utilizan porque desconocen su utilidad teórica y práctica. De este análisis surgió el segundo elemento teórico: "Pensamiento crítico y aprendizaje basado en problemas mediante el uso de la estrategia TAC".

3. El Componente Conceptual, fue producto de las respuestas que dieron los entrevistados 2, 8, 9, 1, 2. Aquí se puso de manifiesto que los docentes a pesar de no tener las competencias para utilizar las estrategias TAC, consideran que son importantes. Señalaban que sólo conocen lo que han escuchado de sus compañeros, en cuanto a que estas son estrategias alternativas emergentes para la construcción y generación del conocimiento. Sin embargo, piensan que para ello se requiere que los docentes tengan las bases teóricas que fundamentan la TAC.

Manifestaron, además, que las TAC son herramientas que permiten no sólo aprender, sino aprender a aprender en apoyo al quehacer pedagógico de los docentes. Otros plantearon que lo importante es saber utilizar las tecnologías, ya que ellas permiten dinamizar las clases, orientar la toma de decisiones y mejorar la calidad educativa. El elemento teórico producto de la interpretación de esta categoría fue "La construcción del conocimiento en el proceso de enseñanzaaprendizaje sustentado en las estrategias TAC".

Los hallazgos fueron producto de la recolección de los datos indicados en la Tabla 1 y en su interpretación y sistematización. De allí se derivaron los siguientes elementos teóricos que fundamentan la estrategia TAC: 


\section{Formación por competencias para la consolidación de las TAC}

El desarrollo de las tecnologías emergentes orientadas hacia la generación del conocimiento y trabajo colaborativo, ha producido cambios y modificaciones en los saberes, ante los cuales la educación no puede mantenerse al margen, sino por el contrario, debe hacerlos suyos y acogerlos para los procesos de formación integral de los estudiantes, más aún de aquellos que serán los futuros docentes del país. A este respecto, Vivancos (2008) señala que en un principio se conocieron las herramientas digitales como TIC, pero ellas han progresado en los últimos años para convertirse en TAC; de ahí la importancia de que el docente utilice estrategias TAC en la formación de los estudiantes. Y ello implica para el docente formarse por competencias y superar el rol de transmisor de conocimientos y centrar su enseñanza en los estudiantes para que estos orienten sus saberes y los desplieguen a situaciones reales.

Desde esta perspectiva, el docente debe tener una sólida formación en el desarrollo de los contenidos conceptuales, procedimentales y actitudinales e internalicen la tecnología como un medio para generar procesos de aprender a aprender. Al respecto, Mejías (2011) indica que "los hipertextos, los multimedia, Internet, la realidad virtual o la televisión por satélite deben ser comprendidas y aplicadas por los docentes en forma interactiva apoyándose en las telecomunicaciones, la informática y los audiovisuales y su hibridación como son los multimedia" (p. 8), pues a través de ellas se accede a la información de los textos, imágenes y sonidos que son fundamentales en el proceso de formación por competencias.

De igual manera, Chavarría (2004) analiza la importancia de las tecnologías y las concibe como mega-tendencia, ya que esta es "Una corriente de pensamiento y de acción, un comportamiento estable y generalizado hacia el cual va dirigido todo acto educativo con el acompañamiento de los protagonistas ámbito, pues se trata de un denominador común en todas las áreas" (p. 15). Medina-Rivilla y Salvador-Mata (2002), por su parte, centran su atención en la importancia que tiene el saber didáctico para los procesos de formación enfatizando en que las estrategias, recursos o técnicas para el aprendizaje son fundamentales para las actividades presenciales y las de distancia.

También Nofal (2007), señala la importancia de estar al día con los procesos del Emprendimiento y Gestión del Conocimiento Tecnológico (TEC), pues se trata de un proceso "lógico, organizado y sistemático para producir, transferir y aplicar en situaciones concretas una combinación armónica de saberes, experiencias, valores, información contextual y apreciaciones expertas que proporcionan un marco para su evaluación e incorporación de nuevas experiencias e información" (p. 1), de notable valor en las competencias de los docentes. 
En consecuencia, el docente debe contar con las competencias para hacer de las TIC recursos aliados en las experiencias de enseñanza y de aprendizaje, para lo cual se proponen las TAC que permiten a los estudiantes y docentes construir saberes que promuevan la criticidad, independencia y autonomía, que sean críticos y autónomos en sus aprendizajes y puedan no solo generar experiencias y procesos de aprendizaje significativos sino el desarrollo de propuestas didácticas de inserción de las TAC en el aula.

Por lo anterior, en el desarrollo humano hay que considerar las dimensiones cognitiva, corporal, social, comunicativa, ética, lúdica, laboral y espiritual. De ahí que cuando se aborda la formación de competencias hay que considerar que la realización del ser humano se produce cuando se construye la propia identidad, al lado de la proyección hacia los demás y al contexto y, por su puesto, hacia el bienestar social. Entonces, es necesario que dentro de las competencias, se consideren las tecnológicas, que deben incluirse en la formación del docente con el fin de que pueda enfrentar los retos que hoy se presentan en el manejo de las TIC para el proceso de enseñanza.

Por su parte, López y Matesanz (2009) indican que las principales competencias en la sociedad del conocimiento son aprender a conocer, b) aprender a querer y sentir, c) aprender a hacer, d) aprender a convivir, e) aprender a ser, f) aprender sobre el conocer, el querer, el sentir. Estas deberán ser aprehendidas por los docentes para poder asumir los retos que impone el siglo XXI. Lo anterior, significa que la formación por competencias es la base fundamental para aprehender el significado y alcance de las TAC en el proceso de enseñanzaaprendizaje.

En este orden de ideas, se resalta el planteamiento que hace Reig (2012), cuando afirma que en el mundo actual es esencial dirigir los esfuerzos hacia la formación en nuevas competencias y la educación en valores, lo mismo que afirmar el rol de profesores e instituciones en la construcción de sentido en todos los intercambios educativos con las nuevas generaciones.

El Portal Educativo Colombia (2014) propone cinco competencias que son fundamentales para el docente: a) La tecnológica; b), Comunicativa; c) Pedagógica, d) Gestión y; e) Investigativa. Igualmente, Tobón (2013), en cuanto a las competencias, presenta un enfoque socio-formativo, en el cual se asumen como "Una dimensión más de la persona humana, la cual se considera en su integralidad y devenir filogenético y ontogenético, articulando la dimensión biológica con la dimensión psicológica, sociológica y espiritual” (p. 45); es decir, comprende más allá de la capacidad de hacer, en cuanto a lo operativo, puesto que la enfoca de manera global e integradora.

Por ello, la competencia tecnológica busca que la educación se integre con las TIC para mejorar los espacios de enseñanza y aprendizaje; además, fortale- 
ce habilidades para aumentar la capacidad de seleccionar y utilizar de la mejor manera las herramientas tecnológicas. La competencia Comunicativa, muestra que las TIC permiten una conexión de expresión entre estudiantes, docentes e investigadores, entre otros, para relacionarse en espacios virtuales y audiovisuales. La Pedagógica, evidencia que mediante las TIC el docente en su quehacer enriquece el arte de enseñar. La competencia de Gestión educativa, resalta que el uso de la tecnología contribuye a desarrollar los procesos de planificación que son necesarios en el quehacer del docente. La Investigativa, se liga con la de gestión y creación del conocimiento.

Es necesario tener en cuenta que el internet ha sido, en las últimas décadas, el repositorio del conocimiento de la humanidad; por eso, es importante que los docentes tengan la capacidad de utilizar las TAC para transformar el saber y generar nuevos conocimientos.

Ahora, estas competencias deben mirarse en forma integral, pues una depende de la otra y son fundamentales en el accionar del docente en el uso de estrategias tecnológicas. Éstas, se complementan con las señaladas en el proyecto "Estándares de Competencias TIC para Docentes" (Organización de las Naciones Unidas para la Educación, la Ciencia y la Cultura [Unesco], 2008). Según este documento, el docente es la persona que desempeña el papel más importante en la tarea de ayudar a los estudiantes a adquirir las capacidades que les permitan ser: a) competentes para utilizar tecnologías de la información; b) buscadores, analizadores y evaluadores de información; c) solucionadores de problemas y tomadores de decisiones; d) usuarios creativos y eficaces de herramientas de productividad; e) comunicadores, colaboradores, publicadores y productores; f) ciudadanos informados, y g) responsables y capaces de contribuir a la sociedad.

Las competencias indicadas exigen una profunda transformación de la educación y de sus instituciones. Al respecto, dice Davini (2008) que en este siglo signado por la abundancia cognitiva, sociedad conectada y en red se imponen nuevos retos al maestro que debe ser consciente de las nuevas habilidades que implica su rol, el cual no solo es educar sino generar y transformar los conocimientos.

En el logro de las competencias antes indicadas, el docente formador de formadores tiene un papel fundamental, ya que debe contribuir a que los estudiantes adquieran esas capacidades diseñando entornos de aprendizaje con estrategias TAC que sean propicias para lograr aprendizajes colaborativos, significativos y metacognitivos. 


\section{Pensamiento crítico y aprendizaje basado en problemas mediante el uso de estrategias TAC}

Este elemento teórico se fundamenta en dos aspectos centrales: la argumentación y la acción, que se alcanzan mediante el uso de las estrategias TAC. Para ello, el docente debe apoyarse en diversas técnicas y actividades organizadas con la finalidad de lograr en los estudiantes su participación e interacción y, por ende, el desarrollo del pensamiento crítico y la resolución de problemas. Desde esta perspectiva, Reig (2012), plantea que en la actualidad existe una revolución comunicación hacia las TAC (Tecnologías del Aprendizaje y el Conocimiento) y las TEP (Tecnologías del Empoderamiento y la Participación). Por tanto, el gran reto que tienen los docentes formadores de formadores, es el aprendizaje de estrategias TAC que permitan que los estudiantes experimenten situaciones interactivas, resuelvan situaciones problemáticas contextualizadas y desarrollen actividades prácticas para estimular el pensamiento crítico y facilitar la transferencia de conocimientos.

Vivancos (2008), por su lado, expresa que las competencias TAC se alcanzan mediante actividades de búsqueda y tratamiento de información a lo cual, el autor le atribuye el desarrollo de la competencia digital. Esta se adquiere con la práctica educativa que va desde la planificación sistémica de las TIC en la institución educativa y en distintos ejemplos de actividades y estrategias en el aula.

Díaz (2003), plantea que "las estrategias tecnológicas deben ser utilizadas por el docente en forma reflexiva y flexible para promover aprendizajes significativos en los alumnos" (p. 4). Por tanto, deben ser motivadoras para los estudiantes y planificadas por el docente con la intención de que los educandos logren conocimientos; por eso, hay que tomar en cuenta los saberes previos. Por otro lado, Ochoa y Sylva (2014) señalan "que es de vital importancia lograr un verdadero aprovechamiento de las estrategias tecnológicas porque ellas representan un recurso de información que impulsa el aprendizaje más allá de la inserción de contenidos en la red” (p. 341).

Por consiguiente, en este trabajo, se plantea que las TAC son herramientas que se sustentan en diferentes estrategias de carácter formativo tanto para el estudiante como para el profesor y garantizan procesos de aprendizaje autónomos, significativos y metacognitivos. Ello, implica no solo conocerlas sino buscar los caminos para utilizarlas y aplicarlas tomando en cuenta las herramientas tecnológicas, necesidades y perfiles de los estudiantes.

El planteamiento antes indicado, guarda relación con el proyecto de estrategias didácticas del Programa "Todos a Aprender" (PTA), con el cual se busca que los docentes mejoren sus prácticas de aula con el desarrollo de entornos de aprendizajes y estrategias que sean dinámicas, interactivas y que contribuyan a 
complementar el proceso de enseñanza y aprendizaje de los estudiantes y generen, por ende, un aprendizaje significativo.

En consecuencia, los autores de este estudio plantean que existe vinculación entre el uso de estrategias interactivas y colaborativas con el aprendizaje significativo que puedan alcanzar los estudiantes en su proceso formativo. Para ello, se fundamentan en Ausubel (1995) cuando resalta que a) El contenido del aprendizaje tiene que ser potencialmente significativo; b) El estudiante debe poseer en su estructura cognitiva los conceptos utilizados previamente formados de manera que el nuevo conocimiento puede vincularse con el anterior y c) El estudiante requiere adoptar una postura positiva hacia el aprendizaje significativo, mostrando disposición para relacionar el material de aprendizaje con su estructura cognitiva.

Este último aspecto indicado por Ausubel (1995) reafirma la importancia que tiene el material de aprendizaje incluyendo dentro de este las estrategias y los recursos para el proceso didáctico de los docentes. Esto exige que tales estrategias sean planificadas y organizadas, mediante el desarrollo de situaciones prácticas o de solución de problemas que integren las tecnologías en los procesos de enseñanza y aprendizaje y contribuyan al desarrollo de la innovación, la creatividad y la generación de conocimientos.

Algunas de las estrategias, técnicas o recursos que pueden utilizarse en el aula y propiciar aprendizajes significativos son, en entre otras, las siguientes: las redes sociales (Facebook, Twitter, LinkedIn, etc.), aprendizaje por proyectos, mapas conceptuales, búsqueda de información, debates (foros, blogs, e-books, chats, video conferencias, etc.), redes de conceptos, solución de problemas, ensayos, gestor de proyectos, la programación y robótica educativa, la realidad aumentada y geolocalización, Impresión y modelado en 3D. Gamificación y Videojuegos educativos, Plataformas de aprendizaje: Moodle, Chamilo, Edmodo, entre otras. Estas deben ser aprovechadas por todos los docentes analizando sus ventajas y desventajas para expandir el conocimiento y, por consiguiente, para analizar, comprender, representar y resolver problemas complejos y la búsqueda de la interrelación teoría-práctica y desarrollo del pensamiento crítico.

En razón de lo anterior, el docente actual debe ser capaz de utilizar las TAC como estrategias pedagógicas que permitan planificar situaciones de aprendizaje en grupos colaborativos y la realización de actividades diversas que propicien el aprendizaje interactivo y significativo. 


\section{La construcción del conocimiento en el proceso de enseñanza-apren- dizaje sustentado en las estrategias TAC}

Este fundamento teórico parte de la concepción de las TAC como estrategias pedagógicas que favorecen la construcción del conocimiento. Esto significa dar el salto de las TIC, orientadas a producir información, hacia otro estadio que es el conocimiento; para ello, en el aprendizaje se debe plantear procesos de orden superior o metacognitivos, lo cual conduce al logro de un aprendizaje centrado en el contexto y en la búsqueda de una construcción social.

El conocimiento es un proceso complejo, contextualizado, histórico, social y cognitivo lo cual plantea que las estrategias TAC que se utilicen deben garantizar la solución de problemas, mediante el uso de técnicas que contribuyan a desarrollar la creatividad, el pensamiento crítico y la interacción social en el aula. En este concepto, el trabajo se apoya en Vygotsky (1979), quien señala que el desarrollo humano es una construcción que se produce en el intercambio del sujeto con su ambiente social. En este enfoque están conceptos como Nivel de desarrollo Real, Nivel de Desarrollo Potencial y Zona de Desarrollo Próximo. El Nivel de Desarrollo Real está determinado por la capacidad para resolver un problema en forma independiente, El Nivel de Desarrollo Potencial está referido a la capacidad para resolver un problema bajo la mediación o guía de otros sujetos de mayor competencia.

Es decir, el educando puede alcanzar niveles jerárquicos más altos y complicados de conocimiento si cuenta con la ayuda de otra persona; en este caso, el docente o un compañero, de mayor competencia. La Zona de Desarrollo Próximo es la distancia que existe entre los dos niveles (Real y Potencial). Entonces, el docente debe propiciar que el alumno vaya desde su zona de desarrollo real hasta su zona de desarrollo potencial; ello lo hace a través de un proceso mediacional, para lo cual tendrá que organizar el ambiente de aprendizaje y trabajar en él para que la mediación sea efectiva. Para ello, deberá tener las competencias necesarias y utilizar todos los recursos que la tecnología le ofrece para desarrollar un proceso educativo creativo desde el desarrollo potencial de sus estudiantes; con esto se fomenta el desarrollo de procesos cognitivos, tanto los de nivel básico como superiores, porque se promueven procesos de pensamiento.

La teoría vygotskiana, entonces, es pertinente en este contexto y, particularmente, en la presente investigación; esto, porque las estrategias TAC en el proceso de formación de los docentes de la Escuela Normal Superior María Auxiliadora de Cúcuta Colombia, y en cualquier otra, harían que el estudiante pueda pasar a un nivel de desarrollo más avanzado con el apoyo del docente para favorecer la interacción de funciones psicológicas nuevas o la reestructuración de las ya existentes. Al respecto, Mena (2004) refiere que: 
Construir (...) un conocimiento pertinente y representativo exige una prolija combinación entre conocimientos teóricos, proporcionados por la ciencia y la tecnología y conocimientos contextualizados, provenientes de la memoria social de los pueblos que componen la región con sus convicciones, necesidades, expectativas y sueños (p.11).

Es decir, no basta con el conocimiento, sino saber aplicarlo. Por ello, Campos, Gaspar y Cortés (2003), refieren que el uso de la estrategia TAC puede: a) proveer condiciones a los estudiantes para la construcción de conocimiento y transformaciones conceptuales; b) favorecer un anclaje al desarrollo de habilidades analítico-categoriales y estratégico-metodológicas, y c) propiciar un ambiente de aprendizaje activo, reflexivo, crítico y participativo. Así, la construcción de conocimientos significa comprender, analizar, sintetizar, argumentar, aspectos estos que deben estar presentes en la implementación de las estrategias TAC que requieren actividades, técnicas, recursos para que los estudiantes adquieran conocimientos.

Desde esta perspectiva, se plantea que las instituciones educativas orientadas en el uso de esta estrategia deben redefinir el papel de todos los elementos implicados en el acto educativo, estudiantes, docentes, gerentes institucionales modelos pedagógicos y el entorno del aula. Esto significa que la construcción del conocimiento con el uso de las TAC dependerá del acoplamiento de todos los elementos antes indicados, es decir, que la institución como un todo, se oriente en teoría y práctica a gestionar los procesos de cambio y a modificar todo aquello que inhiba el desarrollo del conocimiento.

\section{Conclusiones}

Las TAC son estrategias que favorecen la construcción del conocimiento mediante procesos orientados hacia la resolución de problemas, interpretación, argumentación y análisis. Desde esta perspectiva, se plantea la importancia que tiene para el docente conocer el alcance y significado de su aplicación en el aula, dando un salto paradigmático del concepto tradicional de las TIC como medios de obtener información, con el fin de que los estudiantes alcancen procesos de aprendizaje significativos y metacognitivos.

En el desarrollo del trabajo, se generaron tres elementos teóricos que fundamentan las estrategias TAC: Formación por competencias para la consolidación de las TAC; Pensamiento crítico y aprendizaje basado en problemas mediante el 
uso de la estrategia TAC y La construcción del conocimiento en el proceso de enseñanza-aprendizaje sustentado en las estrategias TAC.

Los elementos teóricos indicados deberán ser reforzados mediante el desempeño del docente en el aula lo cual implicará cambios y modificaciones que, sin duda, contribuirán a buscar otros que garanticen el proceso de aprender a aprender con uso de las TIC, las Tac y las TEP, las cuales posibilitan el desarrollo de procesos cognitivos tales como la ampliación de la zona de desarrollo próximo, mayor empoderamiento, el paso de la interacción a la participación y fortalecimiento del aprendizaje situado, uno de los grandes retos de la educación del siglo XXI. Es por ello que Perrenoud (2009), al referirse a la formación de los formadores y a sus instituciones, dice que las últimas deben trabajar con una visión común que apunte a democratizar el acceso a los saberes, a desarrollar la autonomía de los sujetos, su sentido crítico, sus competencias como actores sociales, su capacidad de construir y defender un punto de vista, tanto de manera individual como colectiva.

Para terminar, se reafirma, que este trabajo forma parte de una tesis doctoral en Educación en la cual se aspira contrastar los resultados hasta este momento con otros aportes e insumos conceptuales y empíricos que podrán ratificar o no el supuesto de que las estrategias pedagógicas TAC son la base para el logro de aprendizajes significativos, creativos y para la generación y construcción de conocimientos.

No hay duda, de que esta temática ha sido ampliamente discutida en conferencias, seminarios, talleres orientados hacia la búsqueda de las estrategias y recursos necesarios para que los estudiantes aprendan a aprender con tecnología (Sancho, 2008; Lozano, 2011; Calderón, 2013); esto autores, resaltan la importancia y necesidad de que los estudiantes recurran a la tecnología para la construcción de sus saberes. Sin embargo, la discusión continúa y se ha venido profundizando debido, principalmente, a las interrogantes que formulan los docentes sobre la necesidad de consolidar las TAC en el aula como estrategias que contribuyan a la generación de conocimientos. Además, por cuanto, en la actualidad se puede evidenciar que algunos docentes continúan enseñando en forma tradicional y pese a que se aspira a instaurar la formación por competencias, aún persiste en muchas instituciones la implementación de un modelo curricular conductista, técnico y lineal que no refleja la formación integral del individuo. 


\section{Referencias}

Ángel, D. (2011). La hermenéutica y los métodos de investigación en ciencias sociales. Estudios filosóficos, 44, 9-37. http://www.scielo.org.co/pdf/ef/n44/ n44a02.pdf [Consultado el 2 de octubre de 2018].

Ausubel, D. (1995). Psicología educativa. Un punto de vista cognoscitivo. México, D. F.: Editorial Trillas.

Calderón, M. (2013). De las tecnologías de la información y la comunicación a las tecnologías del aprendizaje y el conocimiento como mediadoras para desarrollar la creatividad en contextos universitarios. Revista Electrónica Formación y Calidad Educativa, 1(1), 29-40. http://www.refcale.uleam.edu. ec/index.php/refcale/article/view/37/35 [Consultado el 20 de septiembre de 2018 ].

Campos, M. A.; Gaspar, S.; Cortés, L. (2003). Una estrategia de enseñanza para la construcción de conocimiento científico (EDCC). Revista Latinoamericana de Estudios Educativos, XXXIII(3), pp. 93-124.

http://www.redalyc.org/pdf/270/27033304.pdf [Consultado el 20 de septiembre de 2018].

Chavarría, O. (2004). Educación en un mundo globalizado. Retos y tendencias del proceso educativo. México, D. F: Editorial Trillas.

Davini, M. C. (2008). Métodos de enseñanza.: didáctica general para maestros y profesores. Buenos Aires, Argentina: Santillana.

Díaz, F. (2003). Estrategias docentes para un aprendizaje significativo: una interpretación constructivista. México D.F.: Me Graw Hill.

Leal, N. (2015). La triangulación en investigaciones sociales y educativas: Orientaciones Generales [Video]. https://studylib.es/doc/5429684/la-triangulaci\%C3\%B3n-en-el-proceso-investigativo [Consultado el 18 de septiembre de 2018].

López, C.; Matesanz, M. (Eds.) (2009). Las plataformas de aprendizaje. Del mito a la realidad. Madrid, España: Universidad Complutense. 
Lozano, R. (2011). De las TIC a las TAC: tecnologías del aprendizaje y del conocimiento. Anuario ThinkEPI, 5, 45-47. https://recyt.fecyt.es/index.php/ ThinkEPI/article/viewFile/30465/16032 [Consultado el 25 de octubre de 2018 ].

Martínez, M. (2007). El paradigma emergente. Hacia una nueva teoría de la racionalidad científica. Barcelona, España: Gedisa.

Medina-Rivilla, A.; Salvador-Mata, F. (Coords.) (2002). Didáctica General. Madrid, España: Prentice Hall.

Mena, M. (Comp.) (2004). La Educación a Distancia en América Latina. Buenos Aires, Argentina: La Crujía.

Mejías, J. (2011). Incorporación de las TIC a la educación. Santiago de Chile: Isol.

Moya-López, M. (2013). De las TIC a las TAC: la importancia de crear contenidos educativos digitales. Revista DIM, 27, 1-15. http://dim.pangea.org/ revistaDIM27/docs/AR27contenidosdigitalesmonicamoya.pdf [Consultado el 21 de octubre de 2018 ].

Nofal, N. (2007). La gestión del conocimiento como fuente de innovación. Revista Escuela de Administración de Negocios, 61, 77-87. https://journal.universidadean.edu.co/index.php/Revista/article/view/418/412 [Consultado el 20 de septiembre de 2018 ].

Ochoa, I.; Sylva, M. (2014). Competencias básicas en el manejo de las TIC en el desempeño del docente universitario. Revista Ciencias de la Educación, 26(47), 338-353. http://servicio.bc.uc.edu.ve/educacion/revista/47/ art21.pdf [Consultado el 22 de octubre de 2018].

Organización de las Naciones Unidas para la Educación, la Ciencia y la Cultura, Unesco. (2008) Estándares de Competencias TIC para Docentes. http:// eduteka.icesi.edu.co/pdfdir/UNESCOEstandaresDocentes.pdf [Consultado el 27 de septiembre de 2018. 
Perrenoud, P. (2009). La formación de los docentes en el siglo XXI. Revista Tecnología Educativa, XIV(3), 503-523. http://academicos.iems.edu.mx/ci$\mathrm{red} / \mathrm{docs} / \mathrm{tg} /$ macroacademiaquimica/La\%20formacion\%20de\%20los\%20 docentes\%20en\%20el\%20siglo\%20XXI_Perrenoud [Consultado el 22 de octubre de 2018].

Portal Educativo Colombia (2014). Conozca las competencias TIC que deben tener los docentes del siglo XXI. https://www.mineducacion.gov.co/1759/articles-339097_archivo_pdf_competencias_tic.pdf [Consultado el 25 de octubre de 2018].

Reig, D. (2012). Estudiantes, autonomía y aprendizaje aumentado: ¿escuelas y docentes como actores clave para otorgar (les) sentido? Encuentro Internacional de Educación, 2012-2013. https://juandomingofarnos.wordpress.com/2012/11/12/ estudiantes-autonomia-y-aprendizaje-aumentado-escuelas-y-docentes-como-actores-clave-para-otorgarles-sentido-encuentro-internacional-de-educacion-2012-2013/

Rodríguez, G.; Gil, J.; García, E. (1999). Metodología de la investigación cualitativa. Málaga, España: Aljibe.

Rosen, L.; Weil, M. (2000s). Computer Availability, Computer Experience and Technophobia Among Public School Teachers. Computers in Human Behavior, 11(1), 9-31. https://www.sciencedirect.com/science/article/pii/O74756329400018D [Consultado el 13 de octubre de 2018 ].

Sancho, J. (2008). De Tic a Tac, el difícil tránsito de una vocal. Barcelona, España: Universidad de Barcelona.

Segura, M.; Candioti, C.; Medina, C. J. (2007). Las TIC en la educación: Panorama internacional y situación española. Documento básico de la XXII Semana Monográfica de Educación, Madrid: España: Fundación Santillana. [Consultado el 27 de septiembre de 2018].

Simoes, M. (2017). Plan de gestión académica para el uso de las TIC en los profesores de gestión ambiental de la Universidad Bolivariana de Venezuela. San Cristóbal, Venezuela: UPEL-IMPM

Stake, R. (1999). Investigación con estudio de casos. Madrid, España: Morata. 
Suay, J. (2017). Tecnofobia, tecnofilia y pesimismo tecnológico. Valencia, España: Universidad Politécnica de Valencia.

Tobón, S. (2013). Formación integraly competencias. Pensamiento complejo, currículo, didáctica y evaluación. Bogotá, Colombia: ECOE Ediciones.

Vivancos, M. J. (2008). Tratamiento de la información y competencia digital. Madrid, España: Alianza Editorial.

Vygotsky, L. (1979). El desarrollo de los procesos psicológicos superiores. Barcelona, España: Crítica. 